\title{
Squeeze until it hurts
}

Geoff J. Pryde

(Geoff J. Pryde is in the Centre for Quantum Dynamics, Griffith University, Brisbane, 4111, Australia. E-mail: g.pryde@griffith.edu.au)

Subject strapline: Quantum physics

Quantum systems are intrinsically uncertain. By "squeezing" down this uncertainty, physicists can use quantum systems to make better measurements of physical quantities such as distance and time. But if you squeeze too hard, things start to burst out all over the place...

At the leading edge of experimental science, the latest measurement techniques are promising breakthroughs in our understanding of the universe. The ever-improving ability to sense small displacements, for example, is at the heart of projects like LIGO, ${ }^{1}$ which seek to observe the faint space-time ripples of distant supernovas. When technical noise is strongly suppressed, the ultimate limit to the precision of any measurement is set by the quantum uncertainty in the measuring system. But even this quantum uncertainty can be reduced - a technique known as squeezing. On page $x x x$ of this issue, Shalm et al. ${ }^{2}$ show that squeezing down this quantum uncertainty is not as simple as might be expected - too much squeezing actually worsens measurement precision. Fortunately, they also show that it is possible to recover the best precision allowed by the laws of physics by looking at the "over-squeezed" system a different way.

That there is a fundamental limit to measurement precision at all is a purely quantum phenomenon. Consider light, the basis for a suite of sensitive interferometric measurement techniques. In a classical picture, light is a wave whose amplitude and phase - where the wave's peaks and troughs lie - can be specified with infinitesimal precision. In reality, light has much more character - it is made up of indivisible photons that exhibit probabilistic behaviour when forced to decide which quantum state - out of a range of options presented - to be in. Shine a hypothetical classical beam on a beam splitter that reflects $50 \%$ and transmits $50 \%$ of the light, and you know that exactly half goes each way. Put a grainy beam - composed of $\mathrm{N}$ photons, say - on that same beam splitter and it is exactly like tossing a coin $\mathrm{N}$ times. You know that on average each outcome should occur N/2 times, but it may not come out that way on a given trial - there is some uncertainty.

A picture may help to understand squeezing. Imagine a stick lying in a plane (figure 1a), such that its length represents the amplitude of the light and its angle represents the phase. The probabilistic nature of photons smears out the end of the stick, leading to uncertainty in the measurement of phase and amplitude. However, quantum theory tells us we can squeeze this uncertainty "blob" in one direction and it will expand in another direction, just like a water-filled balloon. So, squeezing the uncertainty in amplitude leads to an increased phase uncertainty (figure 1b) - but that's OK if it's just the amplitude that we are trying to measure precisely.

Squeezing itself has been demonstrated many times, ${ }^{3}$ but in the regime where squeezing harder just makes things better and better. It has been predicted that in systems with limited dimensions, however, squeezing harder can actually make things worse. ${ }^{4}$ Shalm et al. $^{2}$ have been able to experimentally investigate this fascinating situation by confining themselves to a very quantum system composed of just three photons, which they mash together into the same region of time, space and frequency spectrum. The quantity they measure is not amplitude or phase, but rather polarization - whether the electric field vector of the light oscillates vertically, horizontally, in a circle, or any combination of the above! These three-photon polarization states are not represented by a stick in a plane, but rather by a stick going from the centre of a sphere to the surface, and the quantum uncertainty is represented by a blob on that surface. It is now apparent what happens when squeezing occurs - although the uncertainty is reduced in one 
direction, the blob starts to wrap around the sphere in the other. Eventually it goes all the way around and meets up - although the threefold symmetry leads to a rather more interesting pattern than one might initially expect (figure 2b). This "oversqueezing", however, is problematic. Rather than reducing the uncertainty in the equatorial direction, the oversqueezing has spread the initial uncertainty into three blobs spaced all around the equator, meaning it is now even more uncertain where the end of the stick is - that is to say, what the light's polarization is.

Does this mean that there is an optimal amount of squeezing - not too little and not too much? If one is limited to detecting all the photons in one bunch - an intensity measurement - then the answer is "yes". But when the photons are counted one by one, Shalm et al were able to reveal the correlations between them that give rise to the three fringes around the equator. As this maximally squeezed state is identical to the highly-entangled "NOON" state proposed for quantum metrology, ${ }^{5}$ there exist known adaptive measurement algorithms to extract the complete information in an efficient way. ${ }^{6}$

So, where does all of this leave us? Shalm et al have elegantly demonstrated the connection between the squeezing of quantum states and highly entangled states, by showing the continuum of quantum states with reduced measurement uncertainty. And this idea doesn't only apply to photons - recently similar effects were observed in an atomic spin system. ${ }^{7}$ So are physicists now the masters of quantum uncertainty? Well, not quite - it remains a difficult proposition to highly squeeze large numbers of quantum systems, and a few photons is a long way from the large entangled states required for practical quantum-enhanced precision measurement applications. But our control of the quantum world is ever improving, and we may one day see optimum-precision measurements with large ensembles. In the meantime, we can look for applications of the squeezed and entangled states we can make. ${ }^{8,9}$ And, of course, we can admire the peculiar beauty and symmetries of the quantum world.

\section{$\underline{\text { References }}$}

${ }^{1}$ www.ligo.caltech.edu

${ }^{2}$ Shalm, L. K., Adamson, R. B. A., and Steinberg, A. M., Nature

${ }^{3}$ Walls, D. F., and G. J. Milburn, 1994, Quantum Optics, (Springer-Verlag Berlin)

${ }^{4}$ Combes, J., and Wiseman, H. M., J. Opt. B: Quant. Semiclass. Opt. 7, 14-21 (2005)

${ }^{5}$ Bouwmeester, D. Nature 429, 139-141 (2004)

${ }^{6}$ Higgins, B. L, Berry, D. W., Bartlett, S. D., Wiseman, H. M. and Pryde, G. J., Nature 450, 393396 (2007)

${ }^{7}$ Chaudhury, S., Merkel, S., Herr, T., Silberfarb, A., Deutsch, A. H., and Jessen, P. S., Phys. Rev. Lett. 99, 163002 (2007)

${ }^{8}$ McKenzie, K., Grosse, N., Bowen, W. P., Whitcomb, S. E., Gray, M. B., McClelland, D. E., and Lam, P. K., Phys. Rev. Lett. 93, 161105 (2004); Goda, K., Miyakawa, O., Mikhailov, E. E., Saraf, S., Adhikari, R., Mckenzie, K., Ward, R., Vass, S., Weinstein, A. J., \& Mavalvala, N., Nature Phys. 4, 472 (2008);

${ }^{9}$ Meyer, V., Rowe, M. A., Kielpinski, D., Sackett, C. A., Itano, W. M., Monroe, C. and Wineland, D. J., Phys. Rev. Lett. 86, 5870-5873 (2001)

\section{Figure captions}


a

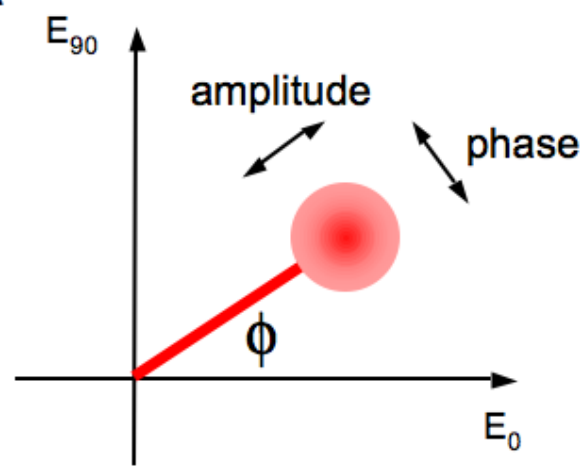

b

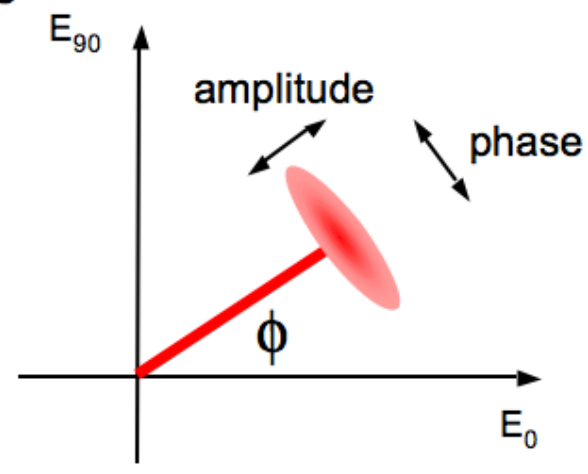

Figure 1. (a) Conceptual representation of the quantum state of light. The length of the red "stick" represents the amplitude of the light, and its angle $\phi$ represents the phase. The "blob" represents the quantum uncertainty in knowing the amplitude and phase. [The blob is usually a Gaussian probability distribution, and the axes $E_{0}$ and $E_{90}$ represent the quadratures of the electric field $E$ at frequency $\omega$ : classically, $\left.E=E_{0} \cos (\omega t)+E_{90} \sin (\omega t)\right]$. (b) A squeezed state. In this example, the amplitude uncertainty is squeezed (decreased) and the phase uncertainty is anti-squeezed (increased).

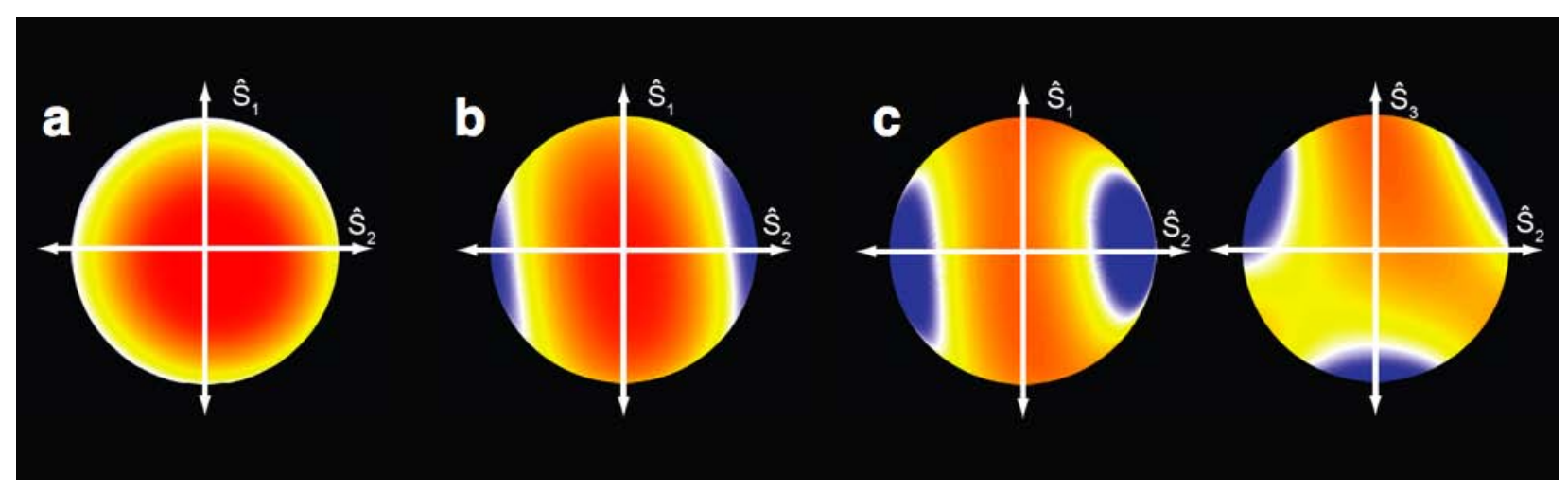

Figure 2. [after Shalm et al. ${ }^{2}$ ] Polarization squeezing of a triphoton. The position of the state on the surface of the sphere describes the polarization state, with the equatorial states representing the degree of $45^{\circ}\left(\mathrm{S}_{2}\right)$ and circular polarization $\left(\mathrm{S}_{3}\right)$. The red and yellow regions represent the uncertainty blob (i.e. the probability distribution of the state vector). The uncertainty in the equatorial direction (i.e. the uncertainty in $45^{\circ}$ polarization) is reduced as the squeezing is increased. (a) An unsqueezed state. (b) As the squeezing is increased, the uncertainty is decreased in the equatorial direction, at the expense of increased uncertainty in the polar direction (which represents the degree of horizontal polarization, $\mathrm{S}_{1}$ ) . (c) In the oversqueezed case, the uncertainty blob completely wraps around the sphere. Although the regions of uncertainty are small in the equatorial direction, there are three of them - as shown in the top view (right) - leading to a large overall uncertainty. 